\title{
Calibration Method for Determining the Physical Location of the Ultrasound Image Plane
}

\author{
Devin V. Amin, Ph.D. ${ }^{1}$, Takeo Kanade, Ph.D ${ }^{1}$., Branislav Jaramaz, Ph.D. ${ }^{2}$, \\ Anthony M. DiGioia III, $\mathrm{MD}^{2}$, Constantinos Nikou, $\mathrm{MS}^{2}$, \\ Richard S. LaBarca, MS ${ }^{1}$, James E. Moody, Jr, $\mathrm{MS}^{2}$
}
${ }^{1}$ Center for Medical Robotics and Computer Assisted Surgery, Carnegie Mellon University, Pittsburgh, PA
${ }^{2}$ Center for Medical Robotics and Computer Assisted Surgery, UPMC Shadyside Hospital, Pittsburgh, PA
\{Devin V. Amin\}da2q@andrew.cmu.edu

\begin{abstract}
This paper describes a calibration method for determining the physical location of the ultrasound (US) image plane relative to a rigidly attached 3D position sensor. A calibrated US probe can measure the 3D spatial location of anatomic structures relative to a global coordinate system. The calibration is performed by aiming the US probe at a calibration target containing a known point ( $1 \mathrm{~mm}$ diameter sphere) in physical space. This point is repeatedly collected at various locations in the US image plane to produce the calibration dataset. An idealized model of the collection process is used to eliminate outliers from the calibration dataset and also to examine the theoretical accuracy limits of this method. The results demonstrate accurate and robust calibration of the $3 \mathrm{D}$ spatial relationship between the US image plane and the $3 \mathrm{D}$ position sensor.
\end{abstract}

\section{Introduction}

Intraoperative ultrasound images allow non-invasive measurement of the bone surface location. This information can be used to perform intraoperative registration to enable computer assisted surgery for minimally invasive procedures that involve limited direct access to the bone surface $[1,4,5,7]$. In order to determine the precise location of the ultrasound (US) image plane, a 3D position sensor (tracker) must be attached to the US probe and the spatial relationship between the tracker and the ultrasound imaging plane must be defined with a calibration routine $[1,2,3,8,9]$. In this study an optical tracker was attached to a removable shell that fit uniquely over the US probe. The calibration data was collected in order to evaluate two hypotheses. The first hypothesis was that the idealized model based outlier elimination process would allow the accuracy of the calibration method to approach the theoretical accuracy limit imposed by the experimental setup. The second hypothesis was that removal and re-attachment of the molded shell would not affect the spatial relationship between the ultrasound image plane and the $3 \mathrm{D}$ position sensor attached to the molded shell. 

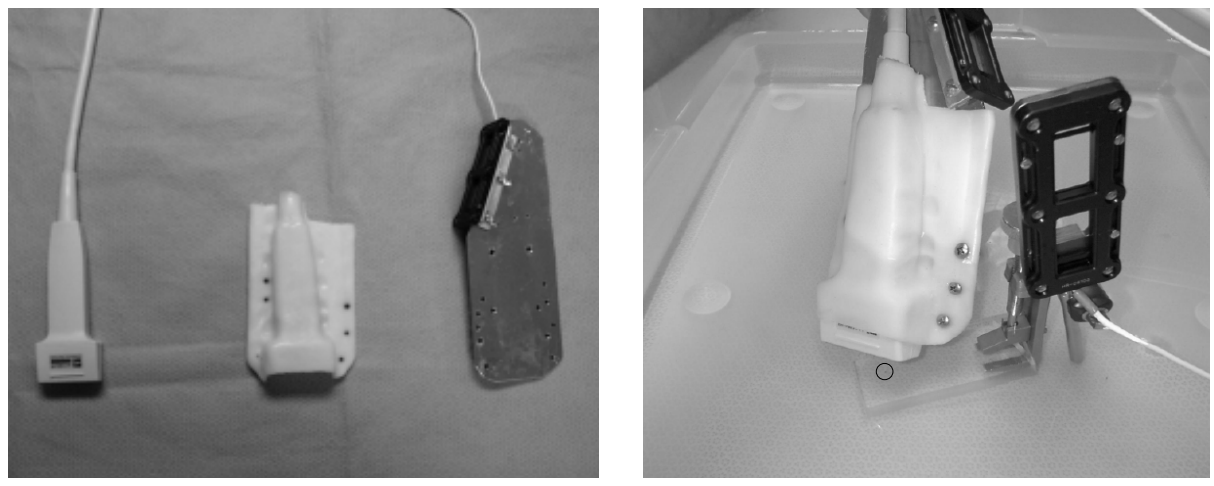

Fig. 1. Left - Ultrasound probe, molded shell and optical position sensor attached to aluminum plate. Right - US probe aimed at fiducial $(1 \mathrm{~mm}$ diameter sphere; located at the center of the circle) on the calibration target.

\section{Methods}

The calibration routine that defines the spatial relationship between the optically tracked molded shell and the US image data is performed once for a given US probe (with fixed imaging parameters such as the depth) and then the removable shell can simply be reattached to the US probe prior to each use in the OR. To attach a 3D position sensor (optical tracker) to the ultrasound probe in a reproducible manner, a two-piece removable molded shell was constructed that fit precisely over the US probe. The first piece was molded to the shape of the US probe using a thermally sensitive plastic (Aquaplast ${ }^{\mathrm{TM}}$, Smith Nephew, $2.4 \mathrm{~mm}$ thickness). The optical tracker was then permanently attached to a rectangular aluminum plate which served as the second piece of the removable shell.

The first step of the calibration process is to determine the physical location of a 1 $\mathrm{mm}$ sphere, termed the US fiducial, using the Optotrak ${ }^{\mathrm{TM}}$ device (Northern Digital Inc., Waterloo, Ontario, Canada). An optically tracked US fiducial tool was calibrated to measure the center of the spherical US fiducial and data was collected to assess the accuracy with which the physical location of the US fiducial could be measured. The physical location was collected 100 times with the US fiducial tool pivoting about the center of the US fiducial; the maximum difference from the mean of the collected locations was less than $0.3 \mathrm{~mm}$. A graphical user interface was used to manually determine the location of the fiducial in each US image (see Fig. 2).

\subsection{Calibration Target}

A calibration target was constructed from by mounting a spherical US fiducial ( $1 \mathrm{~mm}$ diameter steel ball bearing; Applied Industrial, Pittsburgh, PA) to the surface of a rectangular piece of plexiglass. An optical tracker was attached to the calibration target to allow continuous measurement of the physical location of the US fiducial 

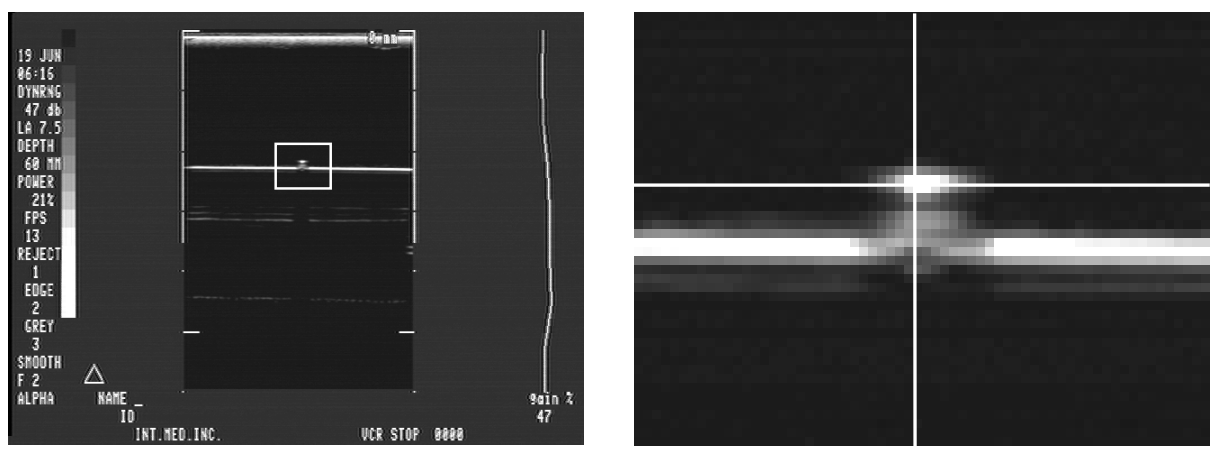

Fig. 2. Left - Ultrasound image with fiducial profile near mid-point of surface contour. Right Close-up with crosshairs indicating the center of the US fiducial in the Image.

(see Fig. 1). The calibration target was submerged in a water medium and images of the US fiducial were obtained.

The ultrasound probe was aimed at the fiducial and images were acquired showing the location of the fiducial within the image (see Fig. 2). The US images were obtained from the video output of the US machine, the position of the fiducial was then specified manually using a graphical user interface, enabling the user to select the $\mathrm{x}$ and $\mathrm{y}$ coordinate of the center of the fiducial marker, as viewed in the US image. The $\mathrm{z}$ coordinate (i.e out of plane direction) was taken to be zero since there was no information available in this direction (i.e. in each calibration image, the US image plane was assumed to intersect the physical center of the US fiducial).

$$
{ }^{\mathrm{US}} \mathrm{P}_{\text {Fiducial }}={ }^{\mathrm{US}} \mathrm{T}_{\mathrm{CB}} \mathrm{x}{ }^{\mathrm{CB}} \mathrm{P}_{\text {Fiducial }}
$$

The physical location of the fiducial, originally collected in the calibration target coordinate frame $\left({ }^{\mathrm{CB}} \mathrm{P}_{\text {Fiducial }}\right)$ was transformed to the coordinate frame of the US probe $\left({ }^{\mathrm{US}} \mathrm{P}_{\mathrm{Fiducial}}\right)$ for each US image using the transform $\left({ }^{\mathrm{US}} \mathrm{T}_{\mathrm{CB}}\right)$ collected by the Optotrack ${ }^{\mathrm{TM}}$ device. The calibration transform $\left({ }^{\mathrm{US}} \mathrm{T}_{\mathrm{IM}}\right)$ was then computed using the two sets of corresponding 3D points (in the image and US coordinate system) with a closed form least squares solution for the absolute orientation [6].

In addition to the six parameters that define the rotation and translation of the calibration transform we must also solve for the scale or ratio of pixels to $\mathrm{mm}$ in the US image. Beginning with an approximate initial value (estimated from the screen) the scale was varied over the range $+/-0.1 \mathrm{pixel} / \mathrm{mm}$ in $0.001 \mathrm{pixel} / \mathrm{mm}$ increments and the scale producing the minimum residual error was used as the scale for the remainder of the calibration studies.

\subsection{Idealized Model Based Outlier Elimination Method}

An idealized model of the calibration process was used to eliminate outlier points from the calibration dataset. The idealized model assumes that the US image plane is infinitely thin (the real US image plane has a thickness in the out of plane direction 

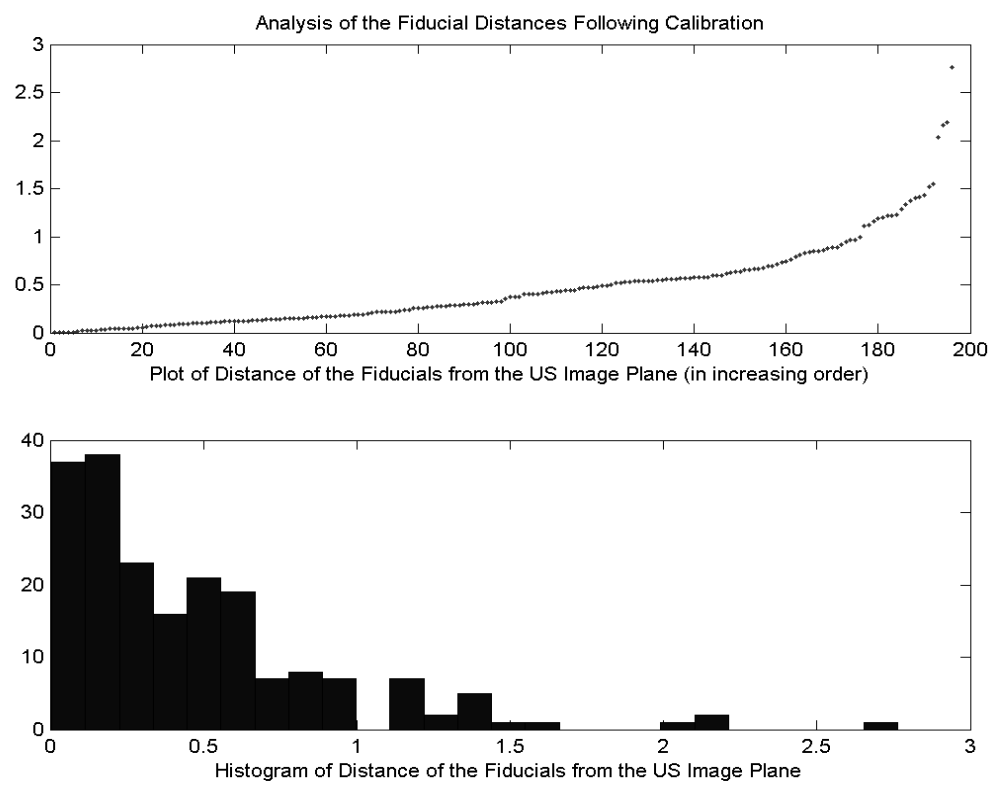

Fig. 3. Analysis of residual distances from the calibrated location of the US image plane.

that varies as a function of distance from the US probe). The physical location of the fiducial can be measured by the US fiducial tool within $0.3 \mathrm{~mm}$ (see section 2). The radius of the US fiducial is $0.5 \mathrm{~mm}$. Therefore, according to the idealized model the maximum distance that a calibration point could be from its closest point on the computed location of the US image plane is $0.8 \mathrm{~mm}(0.5 \mathrm{~mm}$ for the radius of the fiducial and $0.3 \mathrm{~mm}$ for the measurement error). Points further than $0.8 \mathrm{~mm}$ must have some additional source of error contributing to the residual error.

Thus, outliers were defined as points further than $0.8 \mathrm{~mm}$ from the computed location of the US image plane. However, removing one of these outliers from the dataset affects the computation of the US image plane location. Hence, the outliers were eliminated one at a time and after the elimination of each outlier point from the dataset, the calibration transform was recalculated to update all of the residual errors. In this manner, outliers were eliminated until all of the remaining points of the calibration dataset were within $0.8 \mathrm{~mm}$ of the computed location of the US image plane (typically, less than $25 \%$ of the points were eliminated).

\subsection{Theoretical Accuracy}

The idealized model can also be used to evaluate the theoretical accuracy of the calibration method. A simulated calibration dataset was produced using the diameter of the fiducial and the measurement error of the Optotrak device. The image locations ( $\mathrm{z}=0$ for each point) were used as the true locations of the US fiducial, the corresponding location in the US probe coordinate frame was then produced by 

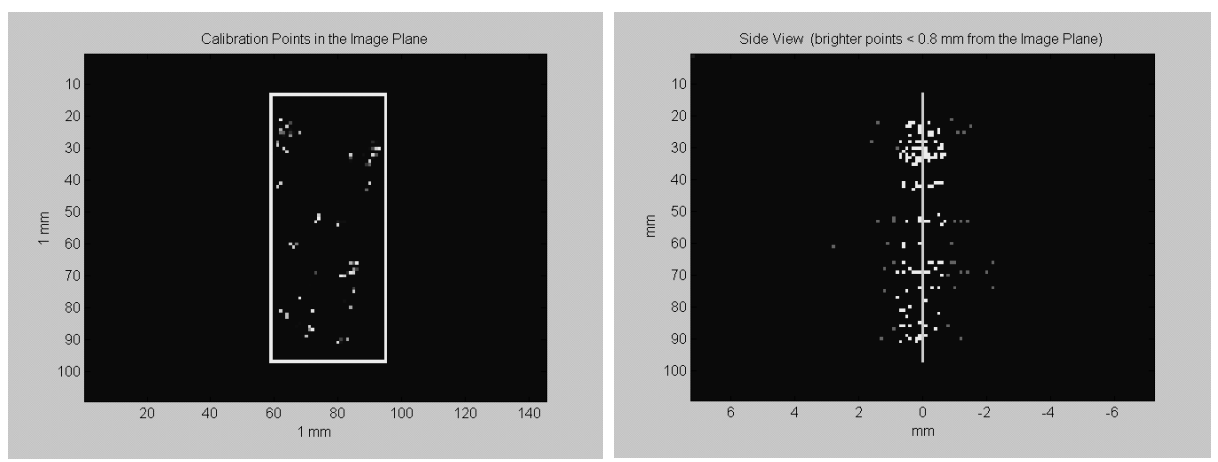

Fig. 4. Left - Distribution of calibration points in the US image plane. Right - Side view showing distance of the calibration points from the image plane (note: the horizontal axis has been magnified by a factor of 10 ).

adding a randomly oriented vector (whose length was uniformly distributed in the interval 0.0 to $0.8 \mathrm{~mm}$ ) to the image location and then transforming the points into the US probe coordinate frame. This simulated dataset (100 calibration points) was subjected to the calibration method described above and the results were compared to the results from the collected calibration data. These simulated results demonstrate the limitations on the achievable accuracy imposed by the experimental setup of the calibration protocol.

\subsection{Consistency of the Calibration Following Reattachment of the Molded Shell}

To evaluate whether the spatial relationship between the optical tracker and the US image plane was identical when the molded shell was reattached, two sets of 100 US images were collected. Between the collection of the first and second set of 100 images the molded shell was removed completely from the US probe and then reattached. The first dataset collected, dataset $\mathrm{A}$, was randomly partitioned into two mutually exclusively sets of 50 points to produce dataset $A_{1}$ and $A_{2}$. In a similar manner, the second dataset collected, dataset $\mathrm{B}$, was partitioned to produce dataset $\mathrm{B}_{1}$ and $\mathrm{B}_{2}$.

By comparing the calibration transform obtained from dataset $\mathrm{A}_{1}\left(\right.$ or $\left.\mathrm{A}_{2}\right)$ to either $\mathrm{B}_{1}$ or $\mathrm{B}_{2}$ we can test the hypothesis that the molded shell attaches in a unique and repeatable manner each time. This comparison is called Inter, since we are comparing calibration transforms obtained before and after removal and reattachment of the molded shell. The other comparison, between dataset $\mathrm{A}_{1}$ and $\mathrm{A}_{2}$ (or $\mathrm{B}_{1}$ and $\mathrm{B}_{2}$ ) is called Intra. In both cases (the Intra or the Inter comparison) the outlier removal procedure described above was performed on both datasets prior to the computation of the calibration transforms. Since some variation in the calibration transform would be expected simply from partitioning the dataset, the Intra dataset comparison serves as a control for the variance from partitioning the dataset. If the molded shell did not reattach with an identical spatial relationship between the US probe and the optically 
tracked molded shell, this difference would be observable as an increased difference in the Inter results as compared to the Intra results.

\section{Results}

Prior to performing the calibration studies, the scale was optimized with respect to the residual error and determined to be 4.415 pixels per $\mathrm{mm}$ (for the particular fixed settings on the US machine used to collect the US images). Six parameters (3 rotations and 3 translations) define the calibration transform that specifies the relationship between the optical tracker and the US image plane. The rotational parameters, $\mathrm{Rx}, \mathrm{Ry}$, and $\mathrm{Rz}$ are reported in degrees and the translational parameters $\mathrm{Tx}, \mathrm{Ty}$, and $\mathrm{Tz}$ are reported in millimeters. The results are presented as the absolute differences in the six parameters that define the two calibration transforms being compared.

\subsection{Theoretical Accuracy}

The first results reported are for the theoretical accuracy study. Tables 1 and 2 display the results using the simulated dataset as compared to the real collected calibration data. The differences between the six calibration parameters using the simulated data indicate the accuracy limitations of this particular experimental setup, and thus are referred to as the theoretical accuracy.

\begin{tabular}{|c|c|c|c|c|c|c|}
\hline & Rx & Ry & Rz & Tx & Ty & Tz \\
\hline Real & 0.5394 & 0.1248 & 0.5147 & 0.1422 & 0.3335 & 0.0378 \\
\hline Simulated & 0.1680 & 0.1252 & 0.2089 & 0.1104 & 0.1210 & 0.0525 \\
\hline
\end{tabular}

Table 1. Theoretical Accuracy Study: Average Differences

\begin{tabular}{|c|c|c|c|c|c|c|}
\hline & Rx & Ry & Rz & Tx & Ty & Tz \\
\hline Real & 1.0849 & 0.3329 & 1.4918 & 0.3392 & 0.8126 & 0.1178 \\
\hline Simulated & 0.2946 & 0.3119 & 0.5345 & 0.2292 & 0.2356 & 0.1015 \\
\hline
\end{tabular}

Table 2. Theoretical Accuracy Study: Maximum Differences

\subsection{Consistency of Calibration Following Removal and Reattachment}

The second set of results evaluate the consistency of reattachment of the molded shell to the US probe. Table 3 lists the average differences and table 4 lists the maximum differences between the six calibration parameters over the set of ten trials of random partitioning of the data. Again, the resulting transforms were compared with the absolute differences between the six parameters that define the calibration transforms. The Inter label indicates that one calibration transform was computed from data collected before and the other after removal of the molded shell. The Intra label 
indicated that both calibration transforms were obtained withouth removing the molded shell. The results demonstrated that the calibration transforms obtained using data collected without removing the shell were not significantly different than those obtained using data collected after removal and reattachment of the molded shell.

\begin{tabular}{|c|c|c|c|c|c|c|}
\hline & $\mathrm{Rx}$ & $\mathrm{Ry}$ & $\mathrm{Rz}$ & $\mathrm{Tx}$ & $\mathrm{Ty}$ & $\mathrm{Tz}$ \\
\hline Inter & 0.6096 & 0.2772 & 0.6052 & 0.1804 & 0.2506 & 0.3263 \\
\hline Intra & 0.5394 & 0.1248 & 0.5147 & 0.1422 & 0.3335 & 0.0378 \\
\hline
\end{tabular}

Table 3. Consistency of Calibration Study: Average Differences

\begin{tabular}{|c|c|c|c|c|c|c|}
\hline & $\mathrm{Rx}$ & $\mathrm{Ry}$ & $\mathrm{Rz}$ & $\mathrm{Tx}$ & $\mathrm{Ty}$ & $\mathrm{Tz}$ \\
\hline Inter & 1.2498 & 0.4675 & 1.2468 & 0.4108 & 0.7310 & 0.4072 \\
\hline Intra & 1.0849 & 0.3329 & 1.4918 & 0.3392 & 0.8126 & 0.1178 \\
\hline
\end{tabular}

Table 4. Consistency of Calibration Study: Maximum Differences

\section{Discussion}

The results using the real data were nearly as accurate (within a factor of two on average) as the results using the simulated data indicating that the calibration results are approaching the limit of accuracy attainable given the experimental setup. However, even after application of the outlier elimination method based on the idealized model, the differences using the collected data were still larger than the differences with the simulated data. This may suggest that modeling the residual errors with a uniform distribution underestimates the typical distribution of the residual errors.

Comparisons between the Intra and Inter results for the collected data indicated that the molded shell did in fact reattach in a unique and reproducible manner as there was not any significant difference between the calibration transforms obtained before and after removal and reattachment of the molded shell.

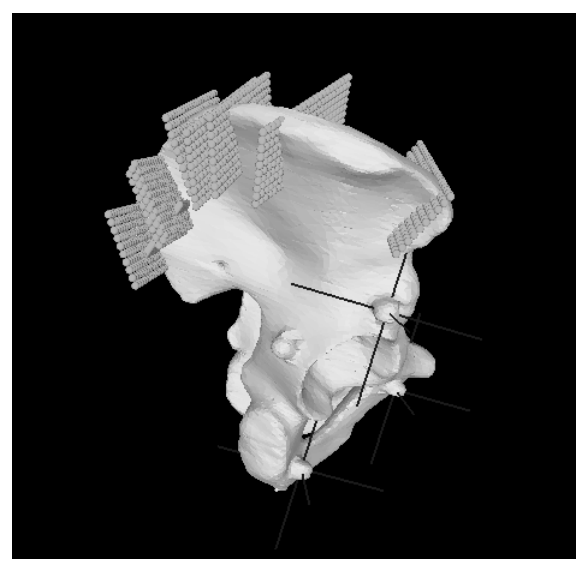

Fig. 5. Set of $2 \mathrm{D}$ ultrasound image planes shown relative to pelvic surface model. 


\section{Conclusion}

The calibration method presented here has several advantages in terms of the accurate and robust determination of the calibration transform. The outlier elimination routine provides a principled method for removing points that are not consistent with the rest of the calibration dataset. Using a large number of calibration points (e.g. 100 or more) allows averaging of the distribution of calibration points to reduce the error associated with the out of plane thickness of the US imaging region. Having the calibration points well distributed throughout the image plane also increases the accuracy by providing a longer baseline to confine the rotational degrees of freedom.

We gratefully acknowlege support for this work from the Medical Robotics and Computer Assisted Surgery Fellowship of the Shadyside Hospital Foundation.

\section{References}

[1] Amin D.V., Ultrasound Registration for Surgical Navigation. PhD Thesis, Carnegie Mellon University, 2001.

[2] Barbe C., Troccaz J., Mazier B., Lavallee S., Using 2.5D Echography in Computer Assisted Spine Surgery. IEEE Engineering in Medicine and Biology Society Proceedings, 1993; 160161.

[3] Blackall J.M., Rueckert D, Mauer C.R., Penney G.P., Hill D.L.G.,Hawkes D.J. An Image Registration Approach to Automated Calibration for Freehand 3D Ultrasound. MICCAI 2000 Proceedings, pp. 462-471, 2000

[4] Carrat L., Tonetti J., Lavallee S. et al, Treatment of pelvic ring fractures: percutaneous computer assisted iliosacral screwing, MICCAI '98 Proceedings, pp. 84-91, 1998

[5] DiGioia A.M., Jaramaz B., Blackwell M., et al. Image Guided Navigation System to Measure Intraoperatively Acetabular Implant Alignment. Clinical Orthopedics and Related Research 355 (1998): 8-23.

[6] Horn B.K.P. Closed form Solution of Absolute Orientation Using Unit Quaternions. Journal of the Optical Society of America A. 4(4):629-642, April, 1987

[7] Ionescu G., Lavallee S., Demongeot J., et al, Automated Registration of ultrasound with CT images: Application to computer assisted prostate radiotherapy and orthopedics, MICCAI '99 Proceedings, 1999

[8] Leotta D.F., Three-dimensional spatial compounding of ultrasound images acquired by freehand scanning: Volume reconstruction of the rotator cuff, $\mathrm{PhD}$ Thesis, University of Washington, 1998

[9] Prager, R., Rohling R., Gee A., Berman L., Rapid calibration for 3D freehand ultrasound. Ultrasound in Medicine and Biology, 24(6):855-869, 1998. 